\title{
An Optical Hydrophone for the Single-Shot Field Measurements of High Power Pressure Pulse Fields
}

\author{
A. JAMShIDI-RAD* AND F. UEBERLE \\ Hamburg University of Applied Sciences, Hamburg 21033, Germany
}

\begin{abstract}
The measurements of ultrasonic fields are often performed using polyvinylidene fluoride (PVDF) membrane hydrophones. Recently, optical hydrophones have gained popularity. Refractive type optical hydrophones are based on the change of refractive index at a glass-water interface when the shockwave passes the sensitive area. A major disadvantage of the optical and the PVDF hydrophones is the fact that they provide a single spot sensitive area, with the result that field measurements need a repositioning of the probe. In this work we introduce a multispot optical hydrophone with six measurement spots, for the single shot field measurement of shockwave fields. We measure a highly stable shockwave source with a calibrated hydrophone and the multi-spot optical hydrophone and we find good agreement for the calculated energy values of the shockwave field (depending on the energy settings $4 \% \pm 1 \%)$.
\end{abstract}

DOI: $10.12693 /$ APhysPolA.127.69

PACS: 06.20.-f, 42.25.Fx, 43.35.Sx, 43.35.Yb

\section{Introduction}

Ultrasound has a wide range of application in medical therapy and diagnostics. The prediction on the efficiency of an ultrasonic medical treatment device and clear statements on the patient safety of a medical ultrasound therapy require the measurement of the pressure field parameters. Despite the longstanding application of medical ultrasound, acoustic field measurements are still a complicated task and not fully understood [1]. Furthermore, for a long time precise measurements of the field parameters in therapeutic ultrasound were not considered as important, as secondary effects such as bleedings and tissue injuries were accepted side effects in lithotripsy [2]. In the early days of lithotripsy the acoustic output of a lithotripter source was characterized by the kilo voltage values of the generator, which does not correlate to the acoustic output of the device. Moreover, to compare two different measurements made at different facilities the measurements need to be standardized by technical requirements. The IEC standard 61846 for instance describes the measurement of lithotripter fields [3]. Although the standard was originally intended for lithotripter fields, it is also in use for the characterization of focused pain therapy sources [4]. The requirements according to the standard are quite challenging. A high dynamic range up to $150 \mathrm{MPa}$, a high bandwidth up to $40 \mathrm{MHz}$ and an active area of $0.2 \mathrm{~mm}$ are some of the toughest requirements [3].

\subsection{State of the art acoustic measurements}

The measurements are carried out by hydrophones in degassed and deionized water. Two main types of hydrophones are available, the piezoelectric and the

* corresponding author; e-mail: abtin.rad@outlook.com piezooptic hydrophones [5]. The polyvinylidene fluoride (PVDF) hydrophones are the sensor of choice when piezoelectric types are requested. Besides the fragile PVDF hydrophones, optical hydrophones are investigated. Their robustness and ease of use as well as the high adhesion of the silica-glass end-face of the sensor to water make the optical hydrophones the sensor of choice for high pressure pulse fields [6]. The first approach for a robust optical hydrophone was the fiber optic hydrophone (FOPH), published by Eisenmenger and Staudenraus in 1988 [7]. The principle of the FOPH is based on the change of light reflectivity at the end-face of a fiber, when the incident wave passes the fiber tip. The pressure wave changes the mass-density of the fluid at the fiber tip, which in turn modulates the optical refractive index. A photodiode converts the reflected laser light into a voltage time signal, which represents the pressure time curve at the location of the fiber tip. The disadvantage of the FOPH is the damageable glass-fiber which tends to break during the measurements, making a complicated re-cutting and repositioning necessary. The light spot hydrophone - LSHD - is based on the same physical principle. The fiber tip is replaced by a ruggedized massive glass block and the sensitive area of the probe is given by the light spot diameter. In case of a local glass fracture the glass block can be displaced without the need of recalibrating or repositioning the sensor [8].

\subsection{New approach of the light spot hydrophone}

Working with single spot hydrophones such as the FOPH the characterization of the spatial pressure distribution needs several measurements at different positions, thus low pulse-to-pulse variation is desirable. This is rarely given with therapeutic sources in particular with pneumatic and electro-hydraulic sources, where the pressure values vary from pulse to pulse over a large interval. To accurately describe the field of such sources the measurements need to be performed in one single shot. For this purpose we developed a novel approach of a 
multi-spot hydrophone using the principle of ultrasound induced refractive index change. The current prototype (Fig. 1) consists of six measurements spots at specific distances to allow the measurements of the $-6 \mathrm{~dB}$ focus width of the majority of lithotripter sources with one single shot.

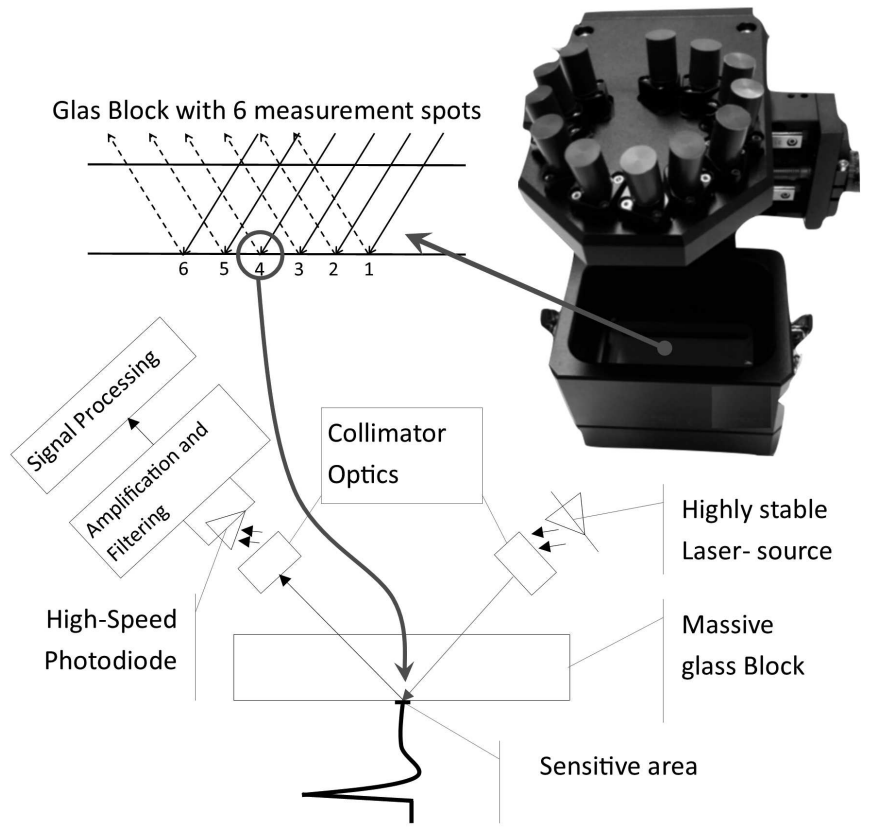

Fig. 1. The principle of the light spot hydrophone using the principle of ultrasound induced refractive index change. In the upper part of the picture the actual prototype of the multi-spot hydrophone with six measurement spots is depicted.

The biggest challenge is the alignment of the focusing optics, to ensure an angle of light incidence under $7^{\circ}$ referred to the glass-surface-normal. This is achieved by a helical arrangement of the focusing optics. The angle of incident light needs to be controlled as the angle decides on the linearity as well as on the sensitivity of the probe. Increasing the angle of incidence increases the sensitivity of the probe, while the linearity is decreased. Based on these dependences a trade-off between the linearity and the sensitivity of the probe is found at $7^{\circ}[8]$.

The signal processing chain consists of a photodiode connected to two different filter and amplifier modules (Fig. 2). The measured light-amplitude-modulation is driven by the sound-wave induced change of the refractive index of the fluid. Thus the reflected light consists of a static (reflection of light in ambient pressure) and dynamic (shockwave driven modulation of the refractive index and hence change of the reflected light at the interface) part. Since the static reflection is several orders higher dynamic reflection, the static signal is separated in a second path.

By having two different amplification paths the requirements for the dynamic range of the amplifier can be

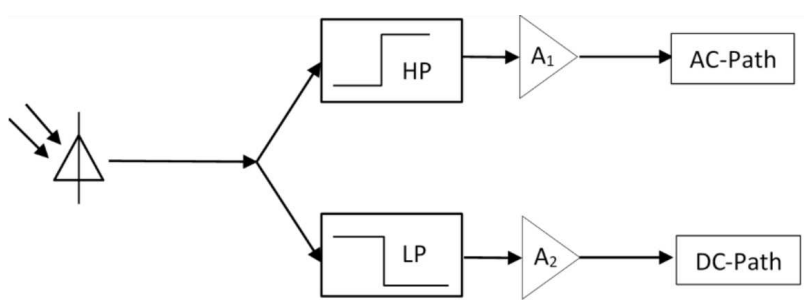

Fig. 2. The signal processing chain of the refractive type hydrophone with two different signal paths: one for the calibration signal (DC-Path) and one for the measurements signal (AC-Path).

reduced and by this the signal-to-noise ratio is improved. The cutoff frequency of the filter has to be chosen very carefully to avoid signal distortions. In a recent paper by Ueberle et al. it has been shown that a high frequency cutoff frequency above $5 \mathrm{kHz}$ could lead to pulse shortening and increased peak tensile (rarefactional) pressure values at high energy settings of the source [4]. For the prototype we choose the amplifier of the fiber optic hydrophone with bandwidth of $100 \mathrm{~Hz}$ up to $40 \mathrm{MHz}$.

\section{Measurement results using the new hydrophone}

To compare the performance of the prototype hydrophone we measure the pressure field of a highly stable piezoelectric shockwave source made by Richard Wolf (EMS Swiss PiezoClast). We compare the measurements done with prototype hydrophone and compare them to calibrated fiber optic hydrophone measurements. The measurements are done according to the IEC standard 61846 in degassed $\left(\mathrm{O}_{2}\right.$ concentration $<2 \mathrm{mg} / \mathrm{L})$ and deionized water at $21^{\circ} \mp 1^{\circ}$. The hydrophones are moved by a custom built $3 \mathrm{D}$ positioning system controlled by a Labview ${ }^{\circledR}$ Program (National Instruments). A HAMEG 1508-2 oscilloscope (150 MHz bandwidth and $1 \mathrm{GSa} / \mathrm{s}$, HAMEG Instruments $\mathrm{GmbH}$, Germany) is used for signal acquisition. The signal processing is performed in Matlab ${ }^{\circledR}$ (MathWorks Inc.) and Microsoft Excel ${ }^{\circledR}$.

We find very good agreement between the measurements using the fiber optic hydrophone and our prototype hydrophone, for all energy settings (Fig. 3a-e) of the transducer.

The pressure time curves match very well in the amplitude domain and time domain. The most important value for medical purpose is the acoustic energy applied to biological tissue. Comparing the energy values calculated of the fiber optic and our multi-spot hydrophone data, we found likewise very good agreement with a maximum difference of $5 \%$. For the spatial pressure distribution we find the same agreement for the pressure values (Fig. 3f), with maximal difference between the fiber-optic measurements of about $4 \%$. However, this comparison is only possible with highly stable source as the piezoelectric source we use in our setup. 

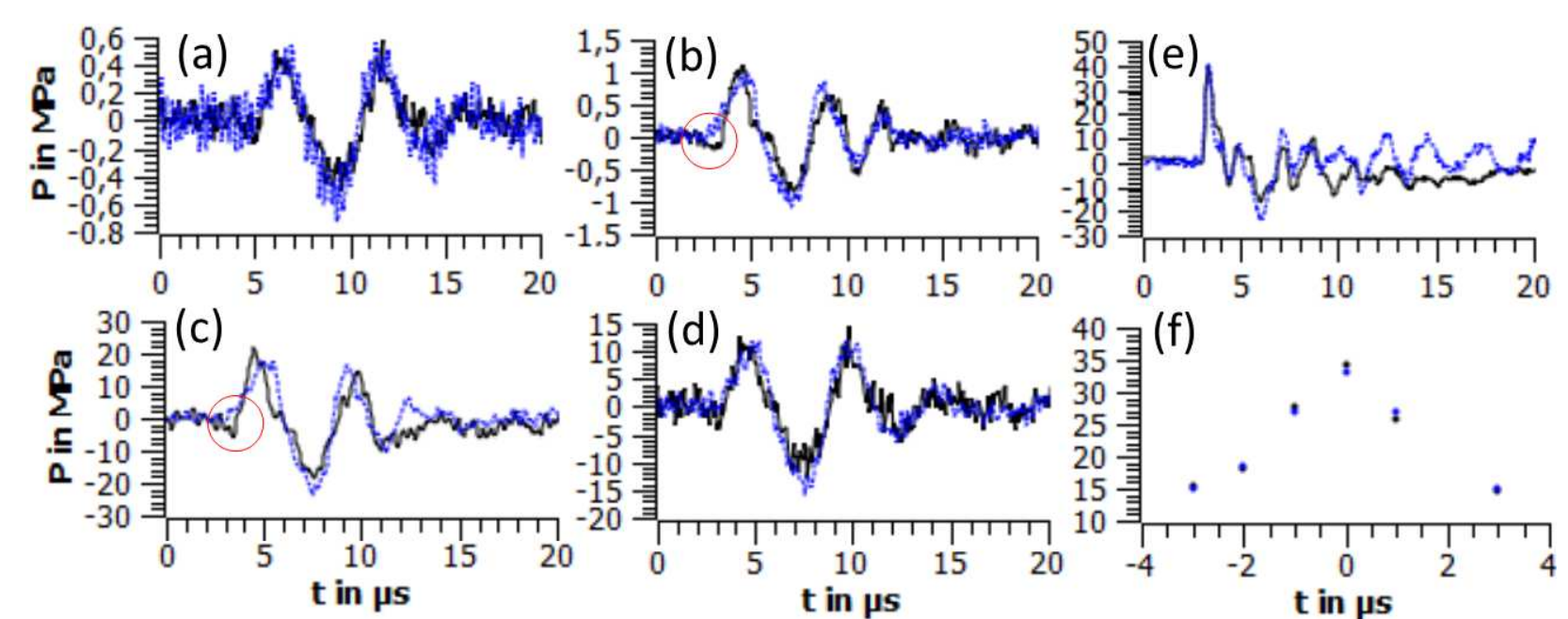

Fig. 3. Measurement of piezoelectric shockwave source (EMS Swiss PiezoClast) at lowest energy setting level 1 (a), level 4 (b), level 10 (c), level 15 (d) and the highest energy setting level 20 (e) using the prototype hydrophone (solid black graph) and the fiber optic hydrophone (blue dotted graph). Single spot measurements are done in the focal plane. In graph (f) the pressure distribution is shown for a single shot measurement using the prototype hydrophone (black dots) and several measurements using the fiber-optic hydrophone (blue dots) at level 17.

\section{Conclusion}

The first experiments done with the multi-spot hydrophone shows good agreement with calibrated hydrophone measurements, making it an adequate probe for the measurement of unstable sources. The maximum pressure that can be measured is dependent on the ruggedness of the glass block and the dynamic range of the amplifier. We could measure a maximum pressure of $40 \mathrm{MPa}$, which is limited to the maximum settings of the source. Based on the better signal-to-noise ratio of the probe it can also be used in diagnostic fields (sensitivity $10 \mathrm{mV} / \mathrm{MPa}$ ). However, taking a closer look at the time-pressure curves we find a rarefactional pulse (of about $1 \%$ of the maximal pressure) before the actual positive pulse (red circle in Fig. 3b and c) which could not be observed in the fiber-optic hydrophones. We assume that the signal distortion is due to bad focusing of the spot at the interface, since investigations on the signal processing chain did not reveal any signal distortions. By adjusting the focusing optics we are able to decrease the signal distortion (Fig. 3d and e), but we were not able to remove the rarefactional pulse completely. Our aim for the next prototype is to remove the signal distortion and improve the signal to noise ratio by customized amplifiers and avalanche photodiodes.

\section{References}

[1] F. Ueberle, A. Jamshidi Rad, J. Phys. 279, 62 (2010).

[2] A. Ilnyckyj, D.H. Hosking, N.M. Pettigrew, C.N. Bernstein, Digest. Dis. Sci. 44, 2485 (1999).

[3] Ultrasonics-Pressure Pulse LithotriptersCharacteristics of Fields, International Electrotechnical Commission, Switzerland 1998.

[4] A. Jamshidi Rad, F. Ueberle, K. Krueger, AIP Rev. Sci. Instrum. 85, 1 (2014).

[5] N. Smith, G.N. Sankin, W.N. Simmons, R. Nanke, J. Fehre, P. Zhong, AIP Rev. Sci. Instrum. 83, 1 (2012).

[6] F. Ueberle, A. Jamshidi Rad, in: Biomed. Tech. 57 S1, 821(2012).

[7] J. Staudenraus, W. Eisenmenger, in: Ultrasonics Symp. IEEE Proc., 1994.

[8] B. Granz, R. Nanke, in: Therapeutic Energy Applications in Urology. Standards and Recent Developments, Eds.: Ch. Chaussy, G. Haupt, D. Jocham, K.U. Köhrmann, D. Wilbert, Thieme, Stuttgart 2005. 